\title{
Parallax and Kinematics of PSR B0919+06 from VLBA Astrometry and Interstellar Scintillometry
}

\author{
S. Chatterjee ${ }^{1}$, J. M. Cordes ${ }^{1}$, T. J. W. Lazio' ${ }^{2}$, W. M. Goss ${ }^{3}$, E. B. Fomalont ${ }^{4}$, \\ J. M. Benson ${ }^{3}$
}

\begin{abstract}
Results are presented from a long-term astrometry program on PSR B0919+06 using the NRAO Very Long Baseline Array. With ten observations (seven epochs) between 1994-2000, we measure a proper motion $\mu_{\alpha}=18.35 \pm 0.06$ mas yr$^{-1}, \mu_{\delta}=86.56 \pm 0.12$ mas yr$^{-1}$, and a parallax $\pi=0.83 \pm 0.13$ mas (68\% confidence intervals). This yields a pulsar distance of $1.21 \pm 0.19 \mathrm{kpc}$, making PSR B0919+06 the farthest pulsar for which a trigonometric parallax has been obtained, and the implied pulsar transverse speed is $505 \pm 80 \mathrm{~km} \mathrm{~s}^{-1}$. Combining the distance estimate with interstellar scintillation data spanning 20 years, we infer the existence of a patchy or clumpy scattering screen along the line of sight in addition to the distributed electron density predicted by models for the Galaxy, and constrain the location of this scattering region to within $\sim 250$ parsecs of the Sun. Comparison with the lines of sight towards other pulsars in the same quadrant of the Galaxy permits refinement of our knowledge of the local interstellar matter in this direction.
\end{abstract}

Subject headings: astrometry_ISM:general—pulsars:general—pulsars:individual (PSR B0919+06)

\section{Introduction}

Since the discovery of pulsars, it has been recognized that the dispersion and scattering of pulsar signals provide unique information about the intervening medium. The distance to most pulsars is estimated using the observed dispersion measure (DM) and a model for the Galactic electron density distribution (?, e.g.)hereafter TC93]TC93. Where available, a model-independent distance from annual trigonometric parallax provides crucial calibration information for this model, as well as allowing the absolute luminosity of the pulsar to be derived.

Pulsar astrometry also provides the observational evidence required to investigate several other questions. Proper motion measurements (especially in conjunction with reliable

\footnotetext{
${ }^{1}$ Cornell University, Ithaca, NY 14853 Contact email: shami@astro.cornell.edu

${ }^{2}$ Naval Research Laboratory, Washington, DC 20375

${ }^{3} \mathrm{NRAO}$, Socorro, NM 87801

${ }^{4}$ NRAO, Charlottesville, VA 22903
}

distance estimates) allow verification of pulsarsupernova remnant associations (?, e.g.)]K98. The inferred speeds of pulsars constrain the minimum asymmetry in supernova core-collapse processes, or other sources of kick velocities. Pulsar population statistics, selection-effect biases, and planetary-extragalactic reference frame ties have also been addressed by astrometry (?, e.g.,)]LL94,CC98,BCR+96.

At present, there are only a handful of modelindependent distances to pulsars. In a recent summary, Toscano et al. (1999a) list nine such objects. In $\S 2$ of this paper, we present results from a longterm astrometry program on PSR B0919+06 using the NRAO Very Long Baseline Array (VLBA). We measure the proper motion and trigonometric parallax for this pulsar, and infer a distance of $\sim 1.2$ $\mathrm{kpc}$ and a transverse speed of $\sim 500 \mathrm{~km} \mathrm{~s}^{-1}$, comparable to the mean population speed.

PSR B0919+06 has a DM of $27.31 \mathrm{pc} \mathrm{cm}^{-3}$ (Phillips and Wolszczan 1992), yielding a mean electron density of $0.023 \mathrm{~cm}^{-3}$ for the interstellar medium (ISM) along this line of sight. However, 
interstellar scintillation (ISS) and scattering of the pulsar signals can be used to extract much more information about the line of sight than simply this mean electron density. In $\S 3$, we review the necessary formalism, and analyze the published data spanning $\sim 20$ years for this pulsar. Our analysis combines data from very long baseline interferometry (VLBI) and ISS, in order to infer the distribution of scattering material along the line of sight. We find the need for extra scattering material in addition to the TC93 distribution; with the assumption that this material is present in the form of a screen or clump with variable scattering strength, we constrain the scren location to the local interstellar medium, within $\sim 250$ parsecs of the Sun.

This analysis, along with DM and distance measurements for other pulsars in the third quadrant of our Galaxy, allows refinement of our knowledge of the local electron density distribution. Besides being a situation of interest in its own right, this analysis also serves as a model for the hybrid VLBI-ISS technique applied to the local ISM.

\section{Obtaining a VLBA Proper Motion and Parallax}

We undertook observations of PSR B0919+06 using the VLBA, over seven epochs (ten observations) from 1994 to 2000 . The details of data acquisition, processing and calibration have been discussed by Fomalont et al. (1999), who presented a preliminary result based on the first four epochs of this dataset. For completeness, we summarize the data reduction procedure here.

\subsection{Phase Referencing and In-Beam Cal- ibration}

In VLBI observations, the visibility phase has to be estimated for each baseline ("fringe fitting") in order to correct for variations in clock offsets, station positions and atmospheric propagation effects. However, fringe fitting is not possible for weak sources, which lack sufficient signalto-noise ratio (SNR) on the short timescales on which the visibility phase varies. Self-calibration enables corrections of unmodeled phase errors, but destroys absolute positional information. As such, astrometric observations must use phasereferencing, where scans are alternated on the (possibly weak) target and a strong nearby calibrator (the "nodding calibrator"). Phase connection across scans on the nodding calibrator allows the visibility phase to be interpolated between the scans on the target, extending the effective coherence time from minutes to hours. This allows astrometry of weak sources, relative to the (assumed fixed) position of the nodding calibrator. This scheme requires limits on both the angular separation between the target and the nodding calibrator, and the cycle time between them. These requiremnts are outlined, for example, by Wrobel at al. (2000), who recommend cycle times ranging from 30 seconds at $43 \mathrm{GHz}$ to 300 seconds at $1.4 \mathrm{GHz}$, with an angular separation $\lesssim 5^{\circ}$.

The negative spectral index of pulsars can be exploited by observations at lower frequencies, where they are stronger. However, the achieved resolution is proportional to the synthesized beam size, and inversely proportional to the SNR. At lower frequencies, the beam is larger, and the system temperatures are generally higher, offseting some of the gain in pulsar signal. Additionally, ionospheric effects become more troublesome at lower frequencies. Our chosen compromise was to observe at L-band (1.4-1.7 GHz), with a typical target-calibrator separation of $5^{\circ}$ and a cycle time of 5 minutes. Even with this strategy, the residual ionospheric errors after phase-referencing can occasionally be large enough to prevent correct phase interpolation and useful astrometry.

Various techniques have been explored to calibrate out differential ionospheric effects, including the use of GPS data (?, e.g.)]RMG+00. For PSR B0919+06, we use an in-beam calibrator, as discussed in detail in Fomalont et al. (1999): there is a faint ( 10 mJy) source, J0922+0638, 12' from the pulsar, within the $\sim 30^{\prime}$ primary beam of a VLBA antenna at $1.4 \mathrm{GHz}$. The pulsar data are correlated twice, once at the pulsar position and once at the position of the in-beam calibrator. We phase-reference the data to the nodding calibrator, self-calibrate the visibility phases so that they are consistent with the visibility function expected for the in-beam calibrator, and transfer these corrections to the pulsar data to carry out the imaging and astrometry.

This procedure has two crucial advantages over ordinary phase-referencing. Phase errors from the different ionospheric electron column densi- 
ties along different lines of sight generally increase with angular separation. Using an in-beam calibrator not only reduces the target-calibrator angular separation from $\sim 5^{\circ}$ to a few arcminutes, but the visibility phase corrections are also derived for the actual times when the pulsar was observed, and thus avoid interpolation over time. This procedure reduces the residual ionospheric errors so that sub-milliarcsecond astrometry is achievable.

\subsection{Imaging and Astrometry}

The VLBA data used here were obtained as part of two different programs, using different nodding calibrators and slightly different frequency setups. The earlier observations (1994-1996) used VLA calibrator $0906+015\left(6^{\circ}\right.$ away from the target) as a nodding calibrator, and a cycle time of 4 minutes on target and 2 minutes on the calibrator. The later observations (1998-2000) used J0914+0245 ( $4^{\circ}$ away) as the calibrator and shortened the cycle time to 3 minutes on target, 2 minutes on calibrator. We also adopted a strategy of snapshot images at two closely spaced epochs (3 to 7 days) in order to verify the consistency of the astrometry, and slightly adjusted the specific observation frequencies (8 independent channels of $8 \mathrm{MHz}$ each) within the $1.4-1.7 \mathrm{GHz}$ band to avoid some known radio frequency interference. During some later epochs, the pulsar was gated at the VLBA correlator, boosting the SNR by $\sim f^{-1 / 2}$, typically a factor of $3-4$, where $f=$ $T_{\text {on }} /\left(T_{\text {on }}+T_{\text {off }}\right)$ is the gate duty cycle.

We note that the changes in observing setup between epochs do not affect the astrometry, since the final pulsar positions are determined relative to the (fixed) position of the in-beam calibrator, which was assumed to be the same at all epochs. The absolute position of the in-beam calibrator, determined with respect to $\mathrm{J} 0914+0245$, is known with a precision of $\sim 10$ mas.

The data were reduced using standard VLB phase-referencing procedures (Beasley \& Conway 1995) using AIPS, the Astronomical Image Processing System. This involved amplitude calibration using the system temperature at each antenna after flagging bad records; a priori phase calibration using pulse calibration tones at the VLBA when available; fringe-fitting to the nodding calibrator; and self-calibration of the in-beam calibrator. The calibration was transferred to the pulsar, and both the in-beam source and the pulsar were imaged. During epochs with a relatively undisturbed ionosphere, the in-beam source was observed to be point-like before self-calibration. Self-calibration yields a point source image for the in-beam source, whose position is used for relative astrometry of the pulsar. Figure 1 shows one pulsar image from each of the seven different epochs, mosaiced together to illustrate the proper motion.

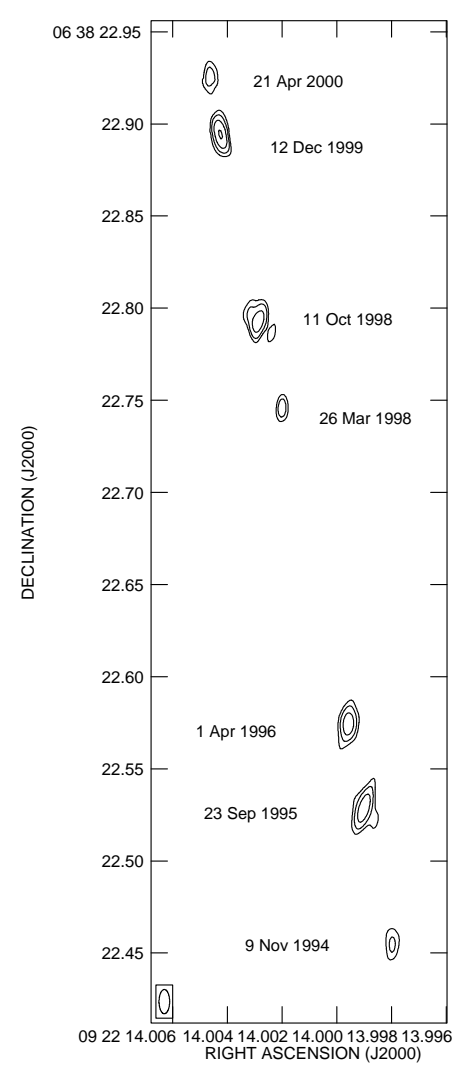

Fig. 1. - Proper motion of PSR B0919+06, depicted as images for a series of epochs. Contours are shown at $5,10,20,40 \sigma$, where $\sigma$ is the RMS map noise. There are no pixels in the map at or below $-5 \sigma$. The period-averaged pulsar flux density varied between 2 and 15 mJy while the RMS noise ranged from 0.1 to $0.9 \mathrm{mJy}$.

The AIPS task JMFIT was used to fit elliptical Gaussians to the images in order to obtain astrometric positions and position errors, as listed in Table 1. The position errors listed for each epoch have three components, which we es- 
timate and add in quadrature: (a) random errors in the position of the in-beam calibrator, estimated as the beam full width at half-maximum $(\mathrm{FWHM}) /(2 \times \mathrm{SNR})$, (b) random errors in the position of the pulsar, estimated as the fitted Gaussian FWHM $/(2 \times \mathrm{SNR})$, and (c) systematic errors in the pulsar position, contributed by the residual ionosphere between the in-beam calibrator and the target, as well as other possibly unknown errors. The deviation of the observed pulsar image from that of a point source convolved with the dirty beam (or point spread function) provides a measure of the residual systematic errors. We take the deconvolved size of the pulsar $\left(\theta_{d}\right.$, which is zero when the image is truly point-like) and use the quantity $\theta_{d} / \sqrt{\left(N_{\text {ant }}-1\right) T_{\text {obs }} / T_{\text {iono }}}$ as an estimate of this last error component. This quantity is not sensitive to the choice of $T_{\text {iono }}$, the ionospheric coherence time, which is empirically determined as $\sim 0.15$ hour. The number of antennas $N_{\text {ant }}$ is usually 10 (or 9 when we lost a VLBA station due to inclement weather) while the observation time $T_{\text {obs }}$ ranged from 1 to 3 hours. The small separation $\left(12^{\prime}\right)$ of the pulsar and the in-beam calibrator implies that any systematic errors in the correlator model (Ma et al. 1998) are negligible, and any residual systematic errors in the astrometry are likely to be small ( $<0.1$ mas).

\subsection{Likelihood Analysis for Astrometric Parameters}

To derive the best fit values and confidence intervals for the parallax and proper motion of PSR B0919+06, we calculate a likelihood function for the parameters that describe the apparent pulsar position as a function of time. These parameters include $\left(\Delta \alpha_{0}, \Delta \delta_{0}\right)$, the offset of the pulsar from an arbitrary assumed position at the reference epoch $(1997.0) ;\left(\mu_{\alpha}, \mu_{\delta}\right)$, the proper motions in right ascension and declination; and $\pi$, the annual trigonometric parallax.

The likelihood function is calculated as follows:

$$
\mathcal{L}=\prod_{i=1}^{N_{\text {data }}} g\left(\left(\alpha_{i}-\hat{\alpha}_{i}\right) / \sigma_{\alpha_{i}}\right) g\left(\left(\delta_{i}-\hat{\delta}_{i}\right) / \sigma_{\delta_{i}}\right)
$$

where

$$
\hat{\alpha_{i}}, \hat{\delta}_{i}=\hat{\alpha}_{i}, \hat{\delta}_{i}\left(\Delta \alpha_{0}, \Delta \delta_{0}, \mu_{\alpha}, \mu_{\delta}, \pi ; t_{i}\right)
$$

are model estimates and $\alpha_{i}, \delta_{i}$ are the observed values of the pulsar coordinates at epoch $t_{i}$ (offsets from the assumed position for 1997.0, as listed in Table 1$), N_{\text {data }}$ is the number of data points (10 in this case), and $g$ is a normalized Gaussian function with zero mean and unit variance.

To obtain the marginal probability distribution of each parameter $\vartheta \in \boldsymbol{\theta}=\left(\Delta \alpha_{0}, \Delta \delta_{0}, \mu_{\alpha}, \mu_{\delta}, \pi\right)$, we calculate the normalized integral of the likelihood function over all other parameters:

$$
f_{\vartheta}(\vartheta)=\frac{\int_{\text {exclude } \vartheta} d \boldsymbol{\theta} \mathcal{L}(\boldsymbol{\theta})}{\int d \boldsymbol{\theta} \mathcal{L}(\boldsymbol{\theta})} .
$$

From the marginal distributions for each parameter, we obtain the best fit (median) values, as well as $68 \%(1 \sigma)$ confidence intervals. These are summarized in Table 2, along with derived estimates for the distance $D$ and transverse velocity $V_{p_{\perp}}$.

Figure 2 shows the residual position offsets after subtracting the best fit proper motion, with the best fit parallax sinusoids overplotted. The marginal probability distribution of the parallax is plotted as an inset in Figure 2. The parallax, $\pi=$ $0.83 \pm 0.13$ mas, differs significantly from Fomalont et al. (1999), who report $\pi=0.31 \pm 0.14$ mas using a subset of the current data set (4 epochs from 1994-1998). It is now apparent that the position errors were underestimated, leading to a parallax estimate $4 \sigma$ displaced from the current result. To test the robustness of the fit reported here, the likelihood analysis was repeated after eliminating data points singly $(1994,2000)$ and in pairs $(1994$ and 2000, both points from October 1998). In all cases, the best fit parameters remained consistent (though with greater uncertainty) with the values for the fit including all data. For example, the $68 \%$ confidence interval for the parallax ranged from $0.81 \pm 0.15$ mas to $0.87 \pm 0.15$ mas, compared to $0.83 \pm 0.13$ mas when using all the data.

We also note that the likelihood of zero parallax is $\sim 8$ orders of magnitude lower than the likelihood of $\pi=0.83$ mas, and the null result is ruled out at over the $6 \sigma$ level. This result implies that even if B0919+06 were twice as far away $(\sim 2.4 \mathrm{kpc})$, the parallax signature of 0.4 mas could have been detected at $3 \sigma$. With a suitable in-beam calibrator and the addition of more sensitive antennas (Arecibo, GBT) to the VLBA, a parallax signature should be detectable to $10 \mathrm{kpc}$ at $5 \sigma$ with $\sim 25$ observations. Beyond this, the variable source structure of calibrators at the 0.1 mas level (Fey, Clegg \& Fomalont 1996) places a basic 
TABle 1

Astrometry Measurements for PSR B0919+06

\begin{tabular}{ccc}
\hline \hline & \multicolumn{2}{c}{ Pulsar Position Offset } \\
\cline { 2 - 3 } Observation Date & East-West (mas) & North-South (mas) \\
\hline $1994.857 \ldots$ & $-45.89 \pm 0.64$ & $-263.20 \pm 1.41$ \\
$1995.728 \ldots$ & $-30.69 \pm 0.33$ & $-189.60 \pm 0.59$ \\
$1996.252 \ldots$ & $-22.20 \pm 0.38$ & $-143.80 \pm 0.76$ \\
$1998.233 \ldots$ & $13.86 \pm 0.31$ & $27.70 \pm 0.73$ \\
$1998.244 \ldots$ & $14.60 \pm 0.32$ & $28.30 \pm 0.77$ \\
$1998.778 \ldots$ & $26.52 \pm 0.89$ & $75.40 \pm 1.02$ \\
$1998.797 \ldots$ & $27.27 \pm 0.97$ & $77.20 \pm 1.35$ \\
$1999.917 \ldots$ & $46.34 \pm 0.20$ & $173.20 \pm 0.52$ \\
$1999.947 \ldots$ & $46.93 \pm 0.17$ & $175.80 \pm 0.38$ \\
$2000.307 \ldots$ & $52.30 \pm 0.49$ & $207.30 \pm 1.03$ \\
\hline
\end{tabular}

${ }^{a}$ Measured from $09^{\mathrm{h}} 22^{\mathrm{m}} 14^{\mathrm{s}} .0011,+06^{\circ} 38^{\prime} 22^{\prime \prime} \cdot 7180$, referenced to in-beam position $09^{\mathrm{h}} 23^{\mathrm{m}} 03.8991$, +06 $38^{\prime} 58^{\prime \prime} .9980$ (J2000.0).

TABLE 2

Astrometric Parameters For PSR B0919+06

\begin{tabular}{lcc}
\hline \hline Parameter & Median value & $1 \sigma$ Error \\
\hline$\mu_{\alpha}\left(\mathrm{mas} \mathrm{yr}^{-1}\right)$ & 18.35 & 0.06 \\
$\mu_{\delta}\left(\mathrm{mas} \mathrm{yr}^{-1}\right)$ & 86.56 & 0.12 \\
$\pi(\mathrm{mas})$ & 0.83 & 0.13 \\
& & \\
$\mathrm{D}(\mathrm{kpc})$ & 1.21 & 0.19 \\
$V_{p_{\perp}\left(\mathrm{km} \mathrm{s}^{-1}\right)}$ & 505 & 80 \\
\hline
\end{tabular}


limit on current astrometric techniques.

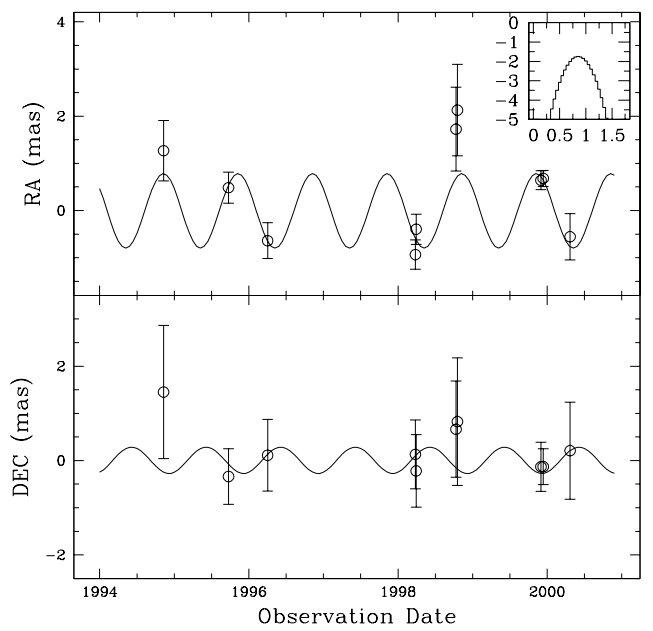

Fig. 2.- Residual position offsets in right ascension and declination after subtracting the proper motion; sinusoids corresponding to the best fit parallax of 0.83 mas are overplotted. Inset: The marginal probability distribution for the parallax, $\log _{10} f_{\pi}(\pi)$ plotted against $\pi$ (mas). A null result is excluded by $\sim 8$ orders of magnitude, or over $6 \sigma$.

\subsection{Transverse Velocity of PSR B0919+06}

From our measurement of the total proper motion $\left(88.48 \pm 0.13\right.$ mas $\left.\mathrm{yr}^{-1}\right)$ and parallax $(0.83 \pm$ 0.13 mas) of B0919+06, we can estimate its transverse velocity to be $505 \pm 80 \mathrm{~km} \mathrm{~s}^{-1}$. This is consistent with the mean pulsar population velocity of $\sim 450 \mathrm{~km} \mathrm{~s}^{-1}$ estimated by Lyne \& Lorimer (1994), as well as the two-component model with characteristic speeds of 175 and $700 \mathrm{~km} \mathrm{~s}^{-1}$ obtained by Cordes \& Chernoff (1998).

\section{Interstellar Scintillation: Data and Analysis}

Electron density fluctuations along the line of sight through the interstellar matter (ISM) scatter radio signals. For pulsars, this phenomenon has been studied extensively through dynamic spectra (intensity variations as a function of time and frequency), whose characteristics allow inferences about the distribution of scattering material along the line of sight. Much of the information in dy- namic spectra can be condensed into two parameters derived from the autocorrelation function $(\mathrm{ACF})$ : the scintillation timescale $\Delta t_{\mathrm{d}}$ (defined as the $1 / e$ width of the ACF intercept on the time lag axis) and the decorrelation bandwidth $\Delta \nu_{\mathrm{d}}$ (the half-width at half maximum of the ACF intercept on the frequency lag axis).

For PSR B0919+06, measurements of these parameters exist over a 20 year timespan, from 1980 to 2000 (Cordes, Weisberg \& Boriakoff 1985; Cordes \& Wolszczan 1986; Bhat, Rao \& Gupta 1999, and unpublished data from Arecibo Observatory in 2000). Cordes \& Wolszczan (1986) observed this pulsar in 1984-85, and report interference fringes due to multipath propagation effects in some of their dynamic spectra, signifying that the ISM had caused multiple imaging of the pulsar, while other epochs show random structure consistent with single-image diffractive scattering. Bhat, Rao \& Gupta (1999) observed this pulsar during 1994, and report gradual and systematic variations in the dynamic spectra, but no fringing events. The derived scintillation parameters for the entire set of data are plotted in Figure 3 (top and middle panels): as expected, these parameters vary significantly with time.

In the analysis that follows, we do not address either the statistical errors in quantifying $\Delta \nu_{\mathrm{d}}, \Delta t_{\mathrm{d}}$ due to the finite number of scintles obtained in each observation or the measurement uncertainties due to the finite number of independent experiments. These errors are adequately represented by the scatter in the data, since we treat each observation independently. Refractive effects may also modify $\Delta \nu_{\mathrm{d}}$ and $\Delta t_{\mathrm{d}}$, particularly in the epochs where multipath effects are important. These effects are difficult to quantify, but we account for their presence in the interpretation.

\subsection{Scintillation and Scattering Formal- ism}

For the analysis, we adopt the theoretical framework of ?)hereafter CR98]CR98, who provide a general treatment of diffractive ISS without assuming any specific scattering geometry (like a thin screen or uniform medium). This generality neccessarily leads to some algebraic complexity, which proves worthwhile in the analysis.

The scintillation speed $V_{\text {ISS }}$ is defined as the 


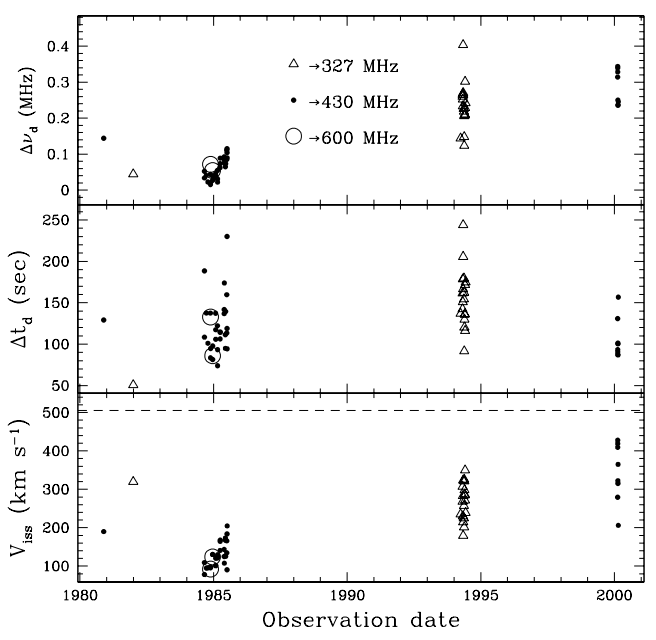

Fig. 3.- Multifrequency scattering parameters for PSR B0919+06: (Top) decorrelation bandwidths $\Delta \nu_{\mathrm{d}}$; (Middle) scintillation timescales $\Delta t_{\mathrm{d}}$; and (Bottom) scintillation speed $V_{\mathrm{ISS}, 5 / 3, \mathrm{u}}$ obtained over 20 years (see text for references). The pulsar speed derived from VLBI parallax and proper motion is plotted with a dashed line.

speed of the ISS diffraction pattern with respect to the observer, which includes contributions from the velocities of the earth and the medium as well as the pulsar. Given values for $D$ (in $\mathrm{kpc}$ ) and observing frequency $\nu$ (in $\mathrm{GHz}$ ), and measurements of $\Delta \nu_{\mathrm{d}}(\mathrm{MHz})$ and $\Delta t_{\mathrm{d}}(\mathrm{sec})$, we obtain $V_{\mathrm{ISS}}$ in $\mathrm{km} \mathrm{s}^{-1}$ (CR98, eq. 13):

$$
V_{\mathrm{ISS}, 5 / 3, \mathrm{u}}=A_{\mathrm{ISS}, 5 / 3, \mathrm{u}} \frac{\sqrt{D \Delta \nu_{\mathrm{d}}}}{\nu \Delta t_{\mathrm{d}}},
$$

where $A_{\mathrm{ISS}, 5 / 3, \mathrm{u}}=2.53 \times 10^{4} \mathrm{~km} \mathrm{~s}^{-1}$, and the subscripts $5 / 3$ and $\mathrm{u}$ denote a Kolmogorov scattering medium that is statistically uniform. The estimated scintillation speed is plotted for the data set in the bottom panel of Figure 3.

To estimate the transverse speed of the pulsar $V_{p_{\perp}}$ from $V_{\text {ISS }}$, we assume that the speeds of the observer and the medium are small compared to the pulsar's; then (CR98, eq. 23):

$$
V_{p_{\perp}}=W_{\mathrm{C}} W_{\mathrm{D}, \mathrm{PM}} V_{\mathrm{ISS}, 5 / 3, \mathrm{u}},
$$

where $W_{\mathrm{C}}$ and $W_{\mathrm{D}, \mathrm{PM}}$ are weighting factors which relate the scintillation pattern speed to the pulsar speed, and rescale the velocity from the uniform
Kolmogorov medium case to the actual medium. $W_{\mathrm{C}}$ depends on the wavenumber spectrum of the medium as well as its distribution (CR98, eq. 18), and its contribution is small (maximum range of 0.9-1.3) unless one assumes solely a thin screen for the distribution of the entire scattering medium. For the remaining analysis, we assume this quantity is unity.

$W_{\mathrm{D}, \mathrm{PM}}$ is a weighting factor determined by the distribution of the scattering material along the line of sight, which is proportional to the coefficient of the electron-density wavenumber spectrum $C_{n}^{2}(s)$ (CR98, Appendix A), where $s$ is the distance measured from the pulsar towards the observer, and varies from 0 to $D$. An exact expression for $W_{\mathrm{D}, \mathrm{PM}}$ is derived for a square law phase structure function in CR98 (eq. 25):

$W_{\mathrm{D}, \mathrm{PM}}(D) \equiv\left[\frac{2 \int_{0}^{D} d s(s / D)(1-s / D) C_{n}^{2}(s)}{\int_{0}^{D} d s(1-s / D)^{2} C_{n}^{2}(s)}\right]^{1 / 2}$.

This expression is only approximately valid for a true Kolmogorov medium, but the approximation is sufficient for our analysis.

\subsection{Hybrid Analysis of VLB and ISS Data}

There are two independent measurements of the transverse velocity of the pulsar, one from the observable VLB proper motion $\mu$, and the other from the measured scintillation parameters $\Delta \nu_{\mathrm{d}}, \Delta t_{\mathrm{d}}$ at an observation frequency $\nu$ :

$$
\begin{aligned}
V_{p_{\perp}} & =\mu D \\
V_{p_{\perp}} & =W_{\mathrm{C}} W_{\mathrm{D}, \mathrm{PM}} A_{\mathrm{ISS}, 5 / 3, \mathrm{u}} \frac{\sqrt{D \Delta \nu_{\mathrm{d}}}}{\nu \Delta t_{\mathrm{d}}} .
\end{aligned}
$$

$W_{\mathrm{D}, \mathrm{PM}}$ depends on the (unknown) distribution of scattering material, as expressed in equation (5), while $D$ is usually known only roughly from dispersion measure-distance models (TC93).

In general, if a parallax distance is unavailable for a pulsar, the different dependencies of $V_{p_{\perp}}$ on $D$ can be used to iterate the two equations $(6,7)$ for a given distribution of scattering material (uniform, exponential, TC93), and thus solve for the distance as well as the transverse velocity. This procedure is especially useful for pulsars well above the Galactic $z$ scale height, where dispersion measure-based models can provide only lower limits on the distance, and parallaxes are not likely to 
be obtained in the forseeable future (as discussed in $\S 2.3)$.

Alternatively, it is possible to refine electron density models by postulating the existence of extra scattering material along the line of sight, in the form of a clump or a screen which contributes increments in dispersion measure $\triangle \mathrm{DM}$ and scattering measure $\Delta \mathrm{SM}$ at a location $D_{s}$. Then a set of constraints on the acceptable values of the screen parameters $\left(\Delta \mathrm{SM}, D_{s}\right)$ as well as the (poorly known) distance $D$ can be derived from this hybrid analysis.

\subsection{Hybrid Analysis using the Parallax Distance for B0919+06}

For PSR B0919+06, the measured parallax distance allows stronger constraints on the scattering geometry. The observation of fringing events for this pulsar, as well as the wide variability in its scintillation parameters (Cordes \& Wolszczan 1986; Bhat, Rao \& Gupta 1999), suggest that this pulsar is viewed through a localized screen or clump of variable scattering strength in addition to a distributed medium. Using only the TC93 electron density model results in an overestimate of the distance to this pulsar, and fails to account for either the observed values of the scattering parameters or their variation with time (Figure 3 ).

We note that large changes in scintillation parameters can be produced with relatively small changes in the average electron density at each epoch. From the definitions of dispersion measure and scattering measure $\left(\mathrm{DM}=\int_{0}^{D} d s n_{e}(s) ; \mathrm{SM}\right.$ $\left.=\int_{0}^{D} d s C_{n}^{2}(s)\right)$, we have:

$$
\begin{aligned}
\Delta \mathrm{DM} & =n_{e, s} \Delta s, \\
\Delta \mathrm{SM} & =C_{n, s}^{2} \Delta s, \\
C_{n, s}^{2} & \propto F_{s} n_{e, s}^{2},
\end{aligned}
$$

where $F$ is a dimensionless "fluctuation parameter" as defined in Taylor \& Cordes (1993), $n_{e}$ is the electron density in the medium, the subscript $s$ designates the values for a screen of thickness $\Delta s$, and $\Delta \mathrm{DM}, \Delta \mathrm{SM}$ are epoch-dependent values. In order to fit the observations, we postulate the existence of a thin patchy screen of scattering material along the line of sight in addition to the TC93 scattering material:

$$
C_{n}^{2}(s)=C_{n, \mathrm{TC} 93}^{2}(s)+\Delta \operatorname{SM} \delta\left(s-D_{s}\right),
$$

where the thin screen contributes an additional scattering measure $\Delta \mathrm{SM}=C_{n, s}^{2} \Delta s$ at a location $s=D_{s}$, represented by the delta function $\delta\left(s-D_{s}\right)$. Recasting equations $(8-10)$ in terms of the differential DM contributed by the screen, and putting in the appropriate scaling factors,

$$
\Delta \mathrm{SM}=\left[(1 / 3)(2 \pi)^{-1 / 3}\right] K_{u} F_{s} \frac{(\Delta \mathrm{DM})^{2}}{\Delta s}
$$

where the scale factor $K_{u}=10.2 \times 10^{-3} \mathrm{~m}^{-20 / 3} \mathrm{~cm}^{6}$ provides the appropriate unit conversion for $\Delta \mathrm{SM}$ in $\mathrm{kpc} \mathrm{m}^{-20 / 3}, \Delta \mathrm{DM}$ in $\mathrm{pc} \mathrm{cm}^{-3}$ and $\Delta s$ in parsecs.

We can rewrite the weighting function $W_{\mathrm{D}, \mathrm{PM}}$ from equation (5) in terms of the TC93 model and this additional scattering screen:

$$
\begin{aligned}
& W_{\mathrm{D}, \mathrm{PM}}(D) \equiv \\
& {\left[\frac{2 \int_{0}^{D} d s(s / D)(1-s / D) C_{n, \mathrm{TC} 93}^{2}(s)+\left(D_{s} / D\right)\left(1-D_{s} / D\right) \Delta \mathrm{SM}}{\int_{0}^{D} d s(1-s / D)^{2} C_{n, \mathrm{TC} 93}^{2}(s)+\left(1-D_{s} / D\right)^{2} \Delta \mathrm{SM}}\right]^{1 / 2} .}
\end{aligned}
$$

We integrate the TC93 model out to the parallax distance derived for this pulsar, which gives $W_{\mathrm{D}, \mathrm{PM}}$ as a function of two screen parameters in equation (13), i.e. the relative scattering strength $\Delta \mathrm{SM} / \mathrm{SM}$, and location $D_{s} / D$.

Additionally, from equations $(6,7)$, we solve for the values of $W_{\mathrm{D}, \mathrm{PM}}$ required to match the scintillation and interferometric velocity estimates (with $W_{\mathrm{C}}$ set to unity):

$$
W_{\mathrm{D}, \mathrm{PM}}=\frac{\mu \nu \Delta t_{\mathrm{d}}}{A_{\mathrm{ISS}, 5 / 3, \mathrm{u}}}\left(\frac{D}{\Delta \nu_{\mathrm{d}}}\right)^{1 / 2}
$$

Thus $W_{\mathrm{D}, \mathrm{PM}}$ is known as a function of $\Delta \mathrm{SM} / \mathrm{SM}$, $D_{s} / D$ (eq. 13 ), and the values of $W_{\mathrm{D}, \mathrm{PM}}$ required for the two estimates of $V_{p_{\perp}}$ (eqs. 6,7) to agree at each observation are specified by equation (14). This information is summarized in Figure 4, where the required values of $W_{\mathrm{D}, \mathrm{PM}}$ are plotted as contours against $\Delta \mathrm{SM} / \mathrm{SM}$ and $D_{s} / D$ on the surface defined by equation (13). The range of values required for the scattering strength and location of the screen (in addition to the TC93 model) can thus be determined.

\subsection{Results from the Hybrid Analysis}

A reasonable assumption is that a turbulent screen may fluctuate in scattering strength on short timescales, while its location varies slowly (if 


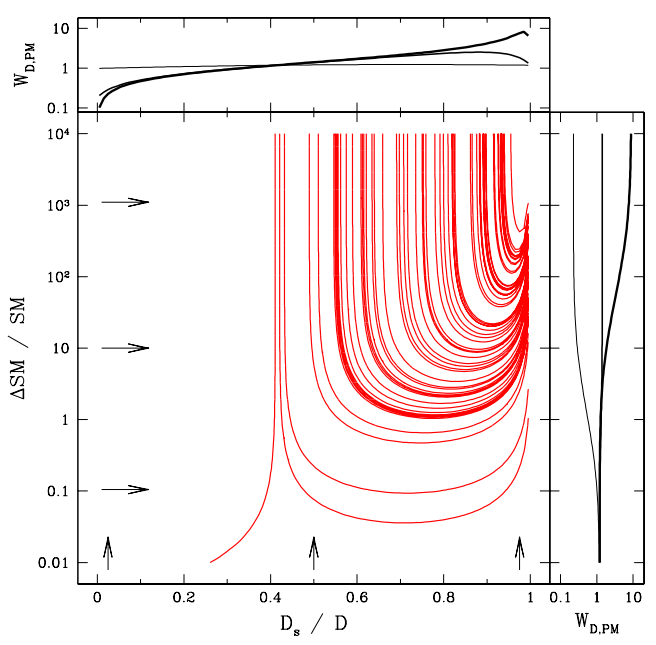

Fig. 4. - $W_{\mathrm{D}, \mathrm{PM}}$ expressed as a function of scattering screen strength $\Delta \mathrm{SM}$ and distance $D_{s}$ from the pulsar towards the observer. The contours represent the value of $W_{\mathrm{D}, \mathrm{PM}}$ required at each epoch shown in Figure 3 for the scintillation speed to match the actual $V_{p_{\perp}}$. Panels show slices through the surface on which contours are drawn, taken at the locations of the arrows. Top panel: $\Delta \mathrm{SM} / \mathrm{SM}=0.1,10,1000$ (thin to thick line); side panel: $D_{s} / D=0.025,0.050,0.975$ (thin to thick line). Assuming a single screen, its location $D_{s} / D$ must be consistent with all of the contours (barring strong refractive scintillation effects), while the range of contours along the $\Delta \mathrm{SM} / \mathrm{SM}$ axis gives the required variation in the scattering strength of the screen.

at all). In that case, the screen must be located at a distance consistent with all of the observed contours in Figure 4, while the spread in the contours along the $\Delta \mathrm{SM} / \mathrm{SM}$ axis gives a measure of the range over which the screen scattering strength has varied over the 20 years spanned by observations. From the figure, consistency with the innermost contours requires $D_{s} / D \gtrsim 0.9$. However, the multiple imaging and fringing events observed during 1986 indicate the occurrence of strong refractive scintillation effects. The current analysis of diffractive scintillation probably overestimates the scattering required during that epoch. Thus the innermost set of contours in Figure 4 may not be useful in locating the screen, and the constraints on the screen can be relaxed to $D_{s} / D \gtrsim 0.8$, with $\Delta \mathrm{SM} / \mathrm{SM}$ ranging from 0.1 to 100 .

For a pulsar distance of $1.2 \mathrm{kpc}$, this result suggests that the scattering screen is within $\sim 240$ parsecs of the Earth, implicating structure within the local ISM. Assuming a screen thickness $\Delta s=10$ parsecs, equation (12) gives an approximate range for $\Delta \mathrm{DM}$ and the fluctuation parameter $F_{s}$ :

$$
10^{-5} \lesssim F_{s}(\Delta \mathrm{DM})^{2} \lesssim 10^{-2}\left(\mathrm{pc} \mathrm{cm}^{-3}\right)^{2} .
$$

Assuming, for example, that $F_{s}=10$ (consistent with values in TC93, Table 2 ), the required range in $\Delta \mathrm{DM}$ is $10^{-3}$ to $3 \times 10^{-2} \mathrm{pc} \mathrm{cm}{ }^{-3}$. For the assumed screen thickness of 10 parsecs, this requires $n_{e, s}$ between $10^{-4}$ and $0.003 \mathrm{~cm}^{-3}$. These values are consistent with the DM variability observed for several nearby pulsars $\left(\Delta \mathrm{DM}_{R M S} \sim\right.$ few $\times 10^{-3}$ pc $\mathrm{cm}^{-3}$ ), including B0919+06 itself, which had a DM variation of $4 \times 10^{-3} \mathrm{pc} \mathrm{cm}^{-3}$ in $1989-1991$ (Phillips and Wolszczan 1992). Of course, in reality, both $F_{s}$ and the screen thickness $\Delta s$ may vary along with changes in electron density, but these values show that the derived constraints on screen location and $\Delta \mathrm{SM} / \mathrm{SM}$ are well within the range of physical possibilities. Additionally, simultaneous measurements of DM variability and changes in ISS parameters can be used to estimate $\Delta s$, which is related to the outer scale size of ISM fluctuations.

\subsection{Interpreting the Screen in terms of the Local ISM}

Combining the analysis for PSR B0919+06 with parallaxes and scattering measurements for other pulsars in the third Galactic quadrant produces a coherent picture of the local ISM in this direction. Measurements of Galactic coordinates $(\ell, b)$, distance, and DM for third-quadrant pulsars are summarized in Table 3 , along with derived values of $n_{e}$, angular separation $\Delta \theta$ and Galactic $z$ height. Toscano et al. (1999a) review the local ISM, and their Figure 2 provides useful background for our discussion. The preceding analysis, and a recent (upward) revision in the distance to PSR B0950+08 (Brisken et al. 2000), allows us to improve upon their discussion for the third Galactic quadrant.

It has long been known that there is an elongated cavity in the local neutral ISM, surrounding 
the Sun and extending several hundred parsecs towards Galactic longitude $\ell=240^{\circ}$. This feature appears to be correlated with the region of low reddening in the Gould Belt, between $\ell=210^{\circ}$ and $255^{\circ}$ (Lucke 1978), and the much-reduced $\mathrm{H}$ I absorption at both high and low latitudes in this longitude range (Paresce 1984). The Local Bubble fills part of this cavity out to a distance of $0.1-0.2 \mathrm{kpc}$ (?, e.g.,)]CR87, though the Bubble boundary is not well defined in this direction. Beyond the Local Bubble, Heiles (1998) has proposed a superbubble, GSH $238+00+09$, along $\ell=238^{\circ}$. This extends from 0.2 to $1.3 \mathrm{kpc}$ from the Sun, and fills another part of the elongated cavity.

It is expected that the region between these two structures has clumps of dense and partially ionized gas, possibly left over from the creation of either (or both) of the bubbles, or from their interaction with each other and their environments. For example, Dupin \& Gry (1998) report the existence of two dense clouds of H II between 40 and $90 \mathrm{pc}$ from the sun, along the line of sight towards $\beta$ Canis Majoris $\left(\ell, b=226^{\circ},-14^{\circ}\right)$, and speculate that these originate in the formation of the Local Bubble. Heiles (1998) postulates "filaments" of warm ionized material on the surface of superbubbles. The scattering properties of various pulsars toward the third Galactic quadrant (Table 3) are consistent with the existence of such a turbulent interface region.

The most striking feature of Table 3 is the difference in $n_{e}$ between the lines of sight to PSRs B0950+08 and B0919+06. Although separated by only 7.8 (35 pc at a distance of $250 \mathrm{pc}$ ), PSR B0919+06 exhibits strong scattering and has undergone episodes of multiple imaging and refractive fringing, while PSR B0950+08 has one of the lowest scattering measures known (Phillips \& Clegg 1992). There are two possible causes for the difference in the properties of these two pulsars.

One possibility is that PSR B0950+08 may be closer than the turbulent interface region. This model requires the distance to the interface region to be larger than $280 \pm 25 \mathrm{pc}$, the (revised) distance to PSR B0950+08, or equivalently, $D_{s} / D<0.8$. We concluded above that $D_{s} / D \gtrsim 0.8$, but given the uncertainties in incorporating the effects of strong refractive scintillation into the estimate for $D_{s} / D$, we can not rule out this possibility. Rather, we take $D_{s} / D \simeq 0.75-0.8$ as a reasonable range for the screen distance.

An alternative possibility is that PSR B0950+08 is within or beyond the turbulent interface region, but the turbulence is concentrated into clumps. In order to cover PSR B0919+06 but not PSR B0950+08, the typical (transverse) scale size of a clump would have to be less than roughly $30 \mathrm{pc}$. This suggestion is consistent with the 10 pc (radial) scale size of the scattering screen that was assumed in our analysis.

The lines of sight to pulsars B0823+26 and J1024-0719 also provide useful information, though the derived constraints are weaker. Both pulsars have mean electron densities along the line of sight comparable to or larger than B0919+06. Both exhibit stronger scattering than B0950+08, particularly B0823+26 which has undergone episodes of strong refractive fringing and multiple imaging (?, e.g.,)]CFC93. J1024-0719 is closer to the Sun than B0950+08, perhaps indicating that B0950+08 is within or beyond the turbulent interface region. However, both $\mathrm{B} 0823+26$ and J1024-0719 are near the edges of the H I cavity. Whether their scattering properties and mean electron densities are due to the "edge" of the $\mathrm{H}$ I cavity or to the interface between the Local Bubble and the GSH $238+00+09$ superbubble is not clear.

We conclude that interstellar scintillometry of PSR B0950+08 and PSR B0919+06 indicates a turbulent interface region between the Local Bubble and the GSH $238+00+09$ superbubble. This turbulent interface region begins $250-300$ pc from the Sun in the direction $(\ell, b) \approx(225,40)$ and possibly contains clumps of scale size $<30$ pc. This result is not inconsistent with the model of Bhat, Gupta \& Rao (1998). Given the height of PSR B0950+08 above the Galactic plane $(\sim 200 \mathrm{pc})$, it is possible that the interface region, and therefore the Local Bubble and the GSH 238+00+09 superbubble, extend to higher Galactic latitudes.

\section{Conclusions}

Parallax distances to pulsars have the prospect of resolving several outstanding questions, both about the pulsar population and about the intervening ISM. In this work, we have presented 
TABle 3

Pulsars in the Third Galactic Quadrant

\begin{tabular}{lcccc}
\hline \hline \multicolumn{1}{c}{ PSR } & B0919+06 & B0950+08 & J1024-0719 & B0823+26 \\
\hline$\ell\left(^{\circ}\right)$ & 225.42 & 228.91 & 251.70 & 196.96 \\
$b\left(^{\circ}\right)$ & +36.39 & +43.70 & +40.52 & +31.74 \\
& & & & \\
$\left.\mathrm{DM}(\mathrm{pc} \mathrm{cm})^{-3}\right)$ & 27.31 & 2.97 & 6.50 & 19.48 \\
$D(\mathrm{kpc})$ & $1.2^{1}$ & $0.28^{2}$ & $<0.23^{3}$ & $0.38^{4}$ \\
$z(\mathrm{kpc})$ & 0.72 & 0.19 & $<0.15$ & 0.20 \\
$n_{e}\left(\mathrm{~cm}^{-3}\right)$ & 0.023 & 0.011 & $>0.029$ & 0.055 \\
$\Delta \theta\left(^{\circ}\right)$ & & & & \\
$\Delta l_{\perp}=D_{s} \Delta \theta(\mathrm{pc})$ & 0 & 7.8 & 21.4 & 23.7 \\
\hline
\end{tabular}

REFERENCES.-Distances from (1) this work; (2) Brisken et al. 2000; (3) Toscano et al. 1999b (upper limit derived from $\mu, \mathrm{P}$, and $\dot{\mathrm{P}}$ ); and (4) Gwinn et al. 1986.

VLBA astrometry on PSR B0919+06, using phase-referenced observations with an in-beam calibrator only $12^{\prime}$ from the pulsar. The use of the in-beam source reduces ionospheric effects and other astrometric errors to the 0.1 mas level and allows sub-milliarcsecond astrometry. With ten observations (seven distinct epochs) distributed over seven years, we derive a proper motion $\mu_{\alpha}=18.35 \pm 0.06$ mas yr$^{-1}, \mu_{\delta}=86.56 \pm 0.12$ mas $\mathrm{yr}^{-1}$, and measure a parallax $\pi=0.83 \pm 0.13$ mas (68\% confidence intervals). This result implies a transverse speed of $505 \pm 80 \mathrm{~km} \mathrm{~s}^{-1}$ at a distance $D=1.21 \pm 0.19 \mathrm{kpc}$, making PSR B0919+06 one of the farthest objects for which a trigonometric parallax has been obtained. The use of an inbeam calibrator with the VLBA at $1.5 \mathrm{GHz}$ can provide trigonometric parallaxes to twice this distance, or even further (to $10 \mathrm{kpc}$ ) with the use of more sensitive antennas and higher frequencies.

For the measured distance of $1.2 \mathrm{kpc}$ and a DM of $27.31 \mathrm{pc} \mathrm{cm}^{-3}$ (Phillips and Wolszczan 1992), the derived mean electron density towards B0919+06 is $0.023 \mathrm{~cm}^{-3}$. This line of sight is along the same Galactic longitude as the superbubble proposed by Heiles (1998), as well as clouds of ionized gas in the local neighbourhood (Dupin \&
Gry 1998), though at a higher Galactic latitude. We use scintillation data spanning 20 years in a hybrid analysis, equating the derived ISS and interferometric speeds of the pulsar in order to constrain the distribution of scattering material along the LOS. We find that excess scattering material is required compared to the standard TC93 model, and assuming that this excess material is confined to a thin screen or clump, we constrain its location to within $\sim 250 \mathrm{pc}$ of the Sun. Comparison with the neighboring lines of sight to pulsars B0950+08, J1024-0719, and B0823+06 indicates that this result is consistent with a turbulent interface region between the Local Bubble and the GSH $238+00+09$ superbubble, which possibly contains clumps of scale size $<30$ pc. We note that using an updated model of the Galactic electron density distribution (Cordes \& Lazio, in preparation) will not materially alter these conclusions.

We thank Dan Stinebring and Maura McLaughlin for access to ISS data from Arecibo Observatory, Don Backer for access to pulsar timing data from the Green Bank $85 \mathrm{ft}$ telescope, and Zaven Arzoumanian and Andrea Lommen for use- 
ful discussions. S.C. gratefully acknowledges the hospitality and assistance of the National Radio Astronomy Observatory, where part of this work was done. NRAO is a facility of the National Science Foundation (NSF) operated under cooperative agreement by Associated Universities, Inc. This work at Cornell was supported in part by NSF grant AST 9819931, and by the National Astronomy and Ionosphere Center, which operates Arecibo Observatory under a cooperative agreement with the NSF. Basic research in radio astronomy at the Naval Research Laboratory is supported by the Office of Naval Research.

\section{REFERENCES}

Bartel, N., Chandler, J. F., Ratner, M. I., Shapiro, I. L., Pan, R. \& Cappallo, R. J. 1996, AJ, 112, 1690

Beasley, A. J. \& Conway, J. E. 1995, ASP Conf. Ser. 82: Very Long Baseline Interferometry and the VLBA, 328

Bhat, N. D. R., Gupta, Y. \& Rao, A. P. 1998, ApJ, 500, 262

Bhat, N. D. R., Rao, A. P. \& Gupta, Y. 1999, ApJS, 121, 483

Brisken, W. F., Benson, J. M., Beasley, A. J., Fomalont, E. B., Goss, W. M., \& Thorsett, S. E. 2000, ApJ, in press

Clegg, A. W., Fiedler, R. L., \& Cordes, J. M. 1993, ApJ, 409, 691

Cordes, J. M. \& Chernoff, D. F. 1998, ApJ, 505, 315

Cordes, J. M. \& Rickett, B. J. 1998, ApJ, 507, 846

Cordes, J. M., Weisberg, J. M. \& Boriakoff, V. 1985, ApJ, 288, 221

Cordes, J. M. \& Wolszczan, A. 1986, ApJ, 307, L27

Cox, D. P. \& Reynolds, R. J. 1987, ARA\&A, 25, 303

Dupin, O. \& Gry, C. 1998, A\&A, 335, 661

Fey, A. L., Clegg, A. W. \& Fomalont, E. B. 1996, ApJS, 105, 299
Fomalont, E. B., Goss, W. M., Beasley, A. J. \& Chatterjee, S. 1999, AJ, 117, 3025

Gwinn, C. R., Taylor, J. H., Weisberg, J. M. \& Rawley, L. A. 1986, AJ, 91, 338

Heiles, C. 1998, ApJ, 498, 689

Kaspi, V. M. 1998, Advances in Space Research, 21,167

Lyne, A. G. \& Lorimer, D. R. 1994, Nature, 369, 127

Lucke, P. B. 1978, A\&A, 64, 367

Ma, C. et al. 1998, AJ, 116, 516

Paresce, F. 1984, AJ, 89, 1022

Phillips, J. A. \& Clegg, A. W. 1992, Nature, 360, 137

Phillips, J. A. \& Wolszczan, A. 1992, ApJ, 385, 273

Ros, E., Marcaide, J. M., Guirado, J. C., Sardón, E. \& Shapiro, I. I. 2000, A\&A, 356, 357

Taylor, J. H. \& Cordes, J. M. 1993, ApJ, 411, 674

Toscano, M., Britton, M. C., Manchester, R. N., Bailes, M., Sandhu, J. S., Kulkarni, S. R. \& Anderson, S. B. 1999, ApJ, 523, L171

Toscano, M., Sandhu, J. S., Bailes, M., Manchester, R. N., Britton, M. C., Kulkarni, S. R., Anderson, S. B. \& Stappers, B. W. 1999, MNRAS, 307, 925

Wrobel, J. M., Walker, R. C., Benson, J. M. \& Beasley, A. J. 2000, VLBA Scientific Memo 24, http://www.aoc.nrao.edu/vlba/html/MEMOS/scimemos.html

This 2-column preprint was prepared with the AAS LATEX macros v5.0. 